\title{
The HV3 Score: A New Simple Tool to Suspect Cognitive Impairment in Multiple Sclerosis in Clinical Practice
}

Muriel Laffon $\cdot$ Grégoire Malandain $\cdot$ Heloise Joly $•$ Mikael Cohen $\cdot$ Christine Lebrun

To view enhanced content go to www.neurologytherapy-open.com

Received: October 17, 2014 / Published online: November 25, 2014

(c) The Author(s) 2014. This article is published with open access at Springerlink.com

\section{ABSTRACT}

Introduction: Cognitive impairment in multiple sclerosis (MS) is common even in the early stages of the disease. Our objective was to improve early detection of cognitive impairment in MS.

Methods: Seventy-five patients with relapsing remitting (RR) MS and 20 controls were enrolled. Two RRMS groups were defined according to their results at the Paced Auditory Serial Addition Test (PASAT). Patients with a $\mathrm{z}$ score below two standard deviations were considered impaired. We quantified T2 and $\mathrm{T} 1$ lesion volumes, and cerebral white and grey matter volumes on a conventional brain magnetic resonance imaging (MRI) scan. Global

Electronic supplementary material The online version of this article (doi:10.1007/s40120-014-0021-x) contains supplementary material, which is available to authorized users.

M. Laffon ( $\varangle) \cdot$ H. Joly · M. Cohen · C. Lebrun Department of Neurology, Pasteur Hospital, University of Nice, 30 Avenue de la Voie Romaine, Nice, France

e-mail: muriel.laffon@gmail.com

M. Laffon · G. Malandain

Institut National de Recherche en Informatique et Automatique (INRIA) Sophia Antipolis, Nice, France brain atrophy was evaluated using the third ventricle (V3) width (in mm). An average brain model was built based on controls and compared with the patient's MRI to quantify regional volumetric changes.

Results: Sixteen (21.3\%) patients with RRMS had low PASAT performance. They had a higher Expanded Disability Status Scale (EDSS) score $(P=0.019)$. T2 and T1 lesion volumes, and grey and white matter volumes were the same in both groups. An enlargement of the V3 width was observed in the low performer group $(P=0.044)$ and V3 width was correlated with the PASAT score $(r=-0.271 ; P=0.021)$. A composite score, named HV3, was obtained by adding the EDSS and V3 width (in $\mathrm{mm}$ ) and correlated with the PASAT $(r=-0.325$; $P=0.006)$. A cutoff HV3 score of over 5.5 identified patients with low PASAT performance, with a positive predictive value of $92.5 \%$ and an accuracy of $70.1 \%$. Focal atrophy was detected in the supplementary motor area, the cingulate gyrus, the right thalamus, and the inferior parietal lobules of patients with lower PASAT performance.

Conclusion: Specific brain morphological changes, including an enlargement of the V3 
width, are associated with low PASAT performance in patients with RRMS. The HV3 score is an additional and complementary tool, accessible in clinical practice, to suspect easily cognitive impairment in patients with RRMS and to better identify patients requiring a complete cognitive assessment.

Keywords: Brain atrophy; Brain MRI; Cognitive impairment; Multiple sclerosis; V3 width

\section{INTRODUCTION}

Cognitive disorders are common in patients with multiple sclerosis (MS) and occur in all MS subtypes with a prevalence of between $40 \%$ and $70 \%$ [1]. They are not only most frequent in progressive forms but are also present at the early stage of the disease [2, 3]. However, their pathophysiology is poorly understood. The cognitive domains most frequently altered include attention, speed of information processing, and working memory [4]. The path to diagnose cognitive disorders is often complicated and requires the use of a battery of tests, including at least $1 \mathrm{~h}$ of cognitive assessment $[5,6]$. Moreover, these batteries can only be implemented by experienced neuropsychologists and are not easy to use in daily neurological practice.

Many magnetic resonance imaging (MRI) parameters are known to be correlated with cognitive impairment in MS, especially global brain atrophy (in particular the width of the third ventricle [V3]) or the basal ganglia volume $[7,8]$. The importance of the involvement of the cortical grey matter volume was more recently demonstrated [9]. Specific brain areas were more atrophied in cognitively impaired patients with relapsing-remitting (RR) MS, especially the thalami, insula, the prefrontal cortex, and regions in the temporo-occipital lobes [10, 11]. In modern connectivity-based approaches, researchers in diffusion-tensor imaging have demonstrated that white matter thalamocortical connections, which constitute the executive system of working memory, were structurally altered in patients with MS [12]. More recently, it has been shown that functional connectivity changes in the thalamocortical network occurred in patients with MS and correlated with cognitive performance [13]. Contradictory data concerned the link between cognitive impairment and $\mathrm{T} 1$ and $\mathrm{T} 2$ lesion loads $[14,15]$.

This study was designed to establish and report correlations between neuropsychological and conventional MRI data. The aim was to improve early detection of cognitive impairment in MS with a simple test based on clinical examination and brain MRI, directly usable during a physician consultation.

\section{METHODS}

\section{Compliance with Ethics Guidelines}

The study was approved by the Nice Ethics Committee. All procedures followed were in accordance with the ethical standards of the responsible committee on human experimentation (institutional and national) and with the Helsinki Declaration of 1975 , as revised in 2000 and 2008. Informed consent was obtained from all patients and controls for being included in the study.

\section{Subjects}

We prospectively enrolled patients with RRMS followed at the tertiary MS center in Nice, France. Patients with a history of psychiatric 
disorders, major depression, or substance abuse were excluded. Demographic data were recorded, including disease duration, first symptoms, and disease-modifying treatment. The level of education and laterality were noted. Disability assessment was based on validated scales: Multiple Sclerosis Functional Composite (MSFC), Expanded Disability Status Scale (EDSS), and Multiple Sclerosis Severity Scale (MSSS). Fatigue was assessed with the validated French fatigue scale "Echelle de Mesure de l'Impact de la Fatigue sur la SEP" (EMIF-SEP) [16].

A group of 20 control patients with no history of neurological or psychiatric diseases was also recruited. Each control patient was matched in age, gender, laterality, and level of education with a subgroup of patients with MS.

\section{Cognitive Assessment}

All patients and controls performed the Paced Auditory Serial Addition Test (PASAT) 3, which was used as a screening test for cognitive assessment [17]. It is a complex test of executive function, working memory, information processing speed, and divided attention. Results were expressed as $\mathrm{z}$ scores to normalize data by age, gender, and level of education by comparison with a standard population [18]. Low performance at this test was defined as a $\mathrm{z}$ score less than two standard deviations (SD) below the normalized PASAT mean.

\section{Brain MRI}

All subjects completed a 1.5 Tesla MRI according to the same protocol. T1-weighted (T1w), T2-weighted spin echo, fluid-attenuated inversion recovery (FLAIR), and $\mathrm{T} 1$ with gadolinium sequences were systematically performed.

SepINRIA software was used to quantify the $\mathrm{T} 2$, T1, and gadolinium lesion loads, and grey and white matter volumes [19]. Global atrophy was indirectly measured on FLAIR sequences by the third ventricle (V3) width, lateral ventricles width, bicaudate ratio, and brain diameter, as described in previous studies [7, 20, 21].

Measures were done manually by two independent evaluators using OsiriX software (Geneva, Switzerland) [22]. Each measure was controlled twice by each evaluator and the average value was conserved for statistical analysis.

To quantify the change in regional volumes, we created a template T1w-MR image by averaging the 3D T1w-MR images from the control group using the methodology presented by Guimond [23]. This average anatomical model of the human brain was then fused with a digital brain atlas from the Montreal Neurological Institute to identify each structure together and with the International Consortium for Brain Mapping (ICBM) atlas to identify white matter tracts. Each patient's MRI was then compared with the average brain model using Jacobian integration to quantify regional volumetric changes. Analysis was conducted using anatomical or functional segmentation (Broadmann areas). As some studies have demonstrated the structures involved in the execution of the PASAT, we specifically focused on the variation in volume of these structures [24].

\section{Statistical Analysis}

Statistical analysis was performed using the XLSTAT $^{\circledR}$ software (Addinsoft, France). For the univariate analysis, the Chi square test was used for categorical data. The Student's $t$ test was 
used for quantitative data. When the distribution was not normal (analysis of variance), the Mann-Whitney test was used. When samples were too small the Fisher's exact test was used for comparison. The level of significance was set at $P<0.05$. Threshold V3 and composite score threshold EDSS + V3 width (HV3 score, chapter results), predictive of low PASAT performance, was calculated using the receiver operating characteristic (ROC) curve and analyzed at the Youden plot. The Pearson's correlation test was performed for continuous variables to determine the presence of a correlation between cognitive performance and clinical and imaging data.

\section{RESULTS}

\section{Demographic and Cognitive Data}

Seventy-five patients with RRMS were enrolled, 57 female and 18 male. All patients were treated with beta interferon. The patients characteristics were mean $( \pm \mathrm{SD})$ age $36.1 \pm$ 9.2 years, mean $( \pm S D)$ level of education $12.8 \pm 2.4$ years, mean $( \pm \mathrm{SD})$ disease duration $6.9 \pm 7.3$ years, median (range) EDSS 1.5 (0-5.5), mean $( \pm \mathrm{SD})$ standardized PASAT $41.65 \pm 12.3$, and mean EMIF-SEP $30.5 \pm 21.2$.

Low performance on the PASAT was detected in $16 / 75$ (21.3\%) of patients with MS. The mean $( \pm$ SD) PASAT score in the MS group with a low PASAT performance was $22.17 \pm 6.27$ against $47.48 \pm 7.92$ in the MS group with normal PASAT performance.

The demographic, clinical, and imaging data of both groups were compared to identify specific parameters associated with a low PASAT performance. There was no difference in age, disease duration, treatment duration or type of treatment, gender, or level of education.
EDSS was significantly higher in the group with low PASAT performance, with a median (range) of $2.0(1-5.5)$ against $1.5(0-5)$ in the group with normal performance $(P=0.019)$. There was no difference in the analysis of the MSSS. There was no difference between the groups for the annualized relapse rate. Patients with MS who demonstrated low performance in the PASAT showed no increase in fatigue. In particular, there was no difference in the cognitive dimension assessed with the EMIF-SEP. All results are presented in Tables 1 and 2 .

\section{Brain MRI Analysis}

\section{MS Patients vs. Controls}

Patients with MS showed a significant enlargement of the V3 width compared with controls $(P=0.0001)$. Similarly, brain width was significantly reduced in the MS group $(P=0.0003)$. There was no difference between the two groups regarding the bicaudate ratio and the width of the lateral ventricles (Tables 3, 4).

\section{Impaired vs. Unimpaired Patients with MS}

There was no significant difference in the T1, $\mathrm{T} 2$, and gadolinium lesion loads, and white matter volume and grey matter volume between the both MS groups.

The width of the V3 was significantly increased in the group with low PASAT performance $(P=0.044)$. There was no difference in the width of the lateral ventricles, the brain diameter, and bicaudate ratio between the two MS groups. The cutoff score of the V3 width predictive of cognitive impairment was over $4.13 \mathrm{~mm}$ with a sensitivity $(95 \% \mathrm{CI})$ of $67.9 \%(55.6 ; 80.1)$ and a specificity (95\% CI) of $68.8 \%(46 ; 91.5)$. The positive predictive value (PPV) was $88.4 \%$. 
Table 1 Demographic characteristics of patients with MS and controls

\begin{tabular}{llll}
\hline Characteristics & Patients with MS $(\boldsymbol{n}=\mathbf{7 5})$ & Controls $(\boldsymbol{n}=\mathbf{2 0})$ & $\boldsymbol{P}$ value \\
\hline Age (years), mean \pm SD & $36.1 \pm 9.2$ & $36.9 \pm 5.8$ & 0.9 \\
Female, $n$ (\%) & $57(76)$ & $11(55)$ & 0.06 \\
Right-handed patients, $n$ (\%) & $60(80)$ & $17(85)$ & 0.61 \\
Level of education (years), mean \pm SD & $12.8 \pm 2.4$ & $13.5 \pm 4$ & 0.47 \\
\hline
\end{tabular}

$M S$ multiple sclerosis, $S D$ standard deviation

Table 2 Demographic characteristics of patients with multiple sclerosis with low PASAT performance compared to normal performers

\begin{tabular}{|c|c|c|c|}
\hline \multirow[t]{2}{*}{ Characteristic } & \multicolumn{2}{|c|}{ PASAT performance } & \multirow[t]{2}{*}{$P$ value } \\
\hline & Impaired & Unimpaired & \\
\hline Sample, $n(\%)$ & $16(21)$ & $59(79)$ & \\
\hline Age (years) & $37.25 \pm 9.45$ & $36.21 \pm 9.1$ & 0.62 \\
\hline Disease duration (years) & $8.6 \pm 7.6$ & $6.66 \pm 7.24$ & 0.35 \\
\hline Level of education (years) & $12.44 \pm 2.87$ & $12.81 \pm 2.22$ & 0.59 \\
\hline Treatment duration (years) & $2 \pm 2$ & $2.36 \pm 3$ & 0.67 \\
\hline History of immunosuppressive therapy, \% & 17 & 20 & 0.8 \\
\hline Annualized relapses rate & $0.8 \pm 0.56$ & $1 \pm 0.93$ & 0.44 \\
\hline EDSS, median (range) & $2.0(1-5.5)$ & $1.5(0-5)$ & 0.019 \\
\hline MSSS & $3.45 \pm 1.9$ & $2.75 \pm 2.2$ & 0.22 \\
\hline \multicolumn{4}{|l|}{ MSFC } \\
\hline 9HPT (s) & $22.43 \pm 5.06$ & $20.05 \pm 5.06$ & 0.102 \\
\hline T25FW (s) & $5.31 \pm 1.64$ & $4.94 \pm 1.41$ & 0.38 \\
\hline Normalized PASAT (/60) & $22.17 \pm 6.27$ & $47.48 \pm 7.92$ & $<0.0001$ \\
\hline EMIF-SEP (/100) & $37.2 \pm 22.4$ & $28.8 \pm 21.3$ & 0.23 \\
\hline Cognitive domain $(/ 100)$ & $38.9 \pm 22.1$ & $27.5 \pm 23.5$ & 0.15 \\
\hline Physical domain (/100) & $45.16 \pm 26.1$ & $37.03 \pm 25.5$ & 0.35 \\
\hline Psychosocial domain (/100) & $37.2 \pm 22.4$ & $24.7 \pm 19.8$ & 0.06 \\
\hline
\end{tabular}

Values are presented as mean \pm standard deviation unless otherwise indicated. Bold indicates a statistically significant difference $(P<0.05)$

9HPT 9 Hole peg test, EDSS Expanded Disability Status Scale, EMIF-SEP Echelle de Mesure de l'Impact de la Fatigue sur la SEP, MSFC Multiple Sclerosis Functional Composite, MSSS Multiple Sclerosis Severity Scale, PASAT Paced Auditory Serial Addition Test, T25FW Timed 25-foot walk 
Table 3 Indirect measurement of global atrophy: comparison between the MS group and control group

\begin{tabular}{lccl}
\hline Measurement & MS group $(n=75)$ & Control group $(n=\mathbf{2 0})$ & $\boldsymbol{P}$ value \\
\hline V3 width $(\mathrm{mm})$ & $\mathbf{4} \pm \mathbf{1 . 7}$ & $\mathbf{2 . 6 5} \pm \mathbf{1 . 1}$ & $\mathbf{0 . 0 0 0 1}$ \\
Bicaudate ratio & $0.115 \pm 0.026$ & $0.124 \pm 0.02$ & 0.136 \\
Lateral ventricles width $(\mathrm{mm})$ & $19.22 \pm 2.95$ & $19.32 \pm 2.3$ & 0.9 \\
Brain diameter $(\mathrm{mm})$ & $\mathbf{1 1 9 . 9 \pm 5 . 1}$ & $\mathbf{1 2 5} \pm \mathbf{6 . 3}$ & $\mathbf{0 . 0 0 0 3}$ \\
\hline
\end{tabular}

Values are presented as mean \pm standard deviation. Bold indicates a statistically significant difference $(P<0.05)$ $M S$ multiple sclerosis, $V 3$ third ventricle

Table 4 Indirect measurement of global atrophy: comparison between the cognitively impaired patients with MS and the unimpaired patients with MS

\begin{tabular}{lccl}
\hline Measurement & \multicolumn{2}{l}{ PASAT performance } & P value \\
\cline { 2 - 4 } & Impaired $(\boldsymbol{n}=\mathbf{1 6})$ & Unimpaired $(\boldsymbol{n}=\mathbf{5 9 )}$ & \\
\hline V3 width $(\mathrm{mm})$ & $\mathbf{4 . 7 3} \pm \mathbf{1 . 4 9}$ & $\mathbf{3 . 7 2} \pm \mathbf{1 . 7 4}$ & $\mathbf{0 . 0 4 4}$ \\
Bicaudate ratio & $0.122 \pm 0.034$ & $0.113 \pm 0.023$ & 0.37 \\
Lateral ventricles width $(\mathrm{mm})$ & $19.18 \pm 3.61$ & $19.18 \pm 2.73$ & 1 \\
Brain diameter $(\mathrm{mm})$ & $119.98 \pm 6.07$ & $119.66 \pm 5.34$ & 0.84 \\
\hline
\end{tabular}

Values are presented as mean \pm standard deviation. Bold indicates a statistically significant difference $(P<0.05)$

$M S$ multiple sclerosis, PASAT Paced Auditory Serial Addition Test, $V 3$ third ventricle

\section{Correlation Analysis}

A correlation analysis between the PASAT score and the available clinical and imaging data was performed. Significant correlation between the PASAT and V3 width $(r=-0.271, P=0.021)$, EDSS $(r=-0.260, P=0.028)$, and 9 Hole Peg Test score $(r=-0.294, \quad P=0.012) \quad$ was demonstrated.

\section{The HV3 Score (Global Handicap Measured by the V3)}

The data were used to define a composite clinical and radiological score easily done in consultation. We statistically analyzed several combinations and selected the one with better correlation with the PASAT score. By adding the EDSS and the V3 width, we obtained significant correlation with the PASAT $(r=-0.325$, $P=0.006$; Fig. 1$)$. This combination of EDSS and V3 width (in mm) was called HV3 (global handicap measured by the V3). The HV3 score correlated better with PASAT than the EDSS or V3 width separately $(r=-0.325, P=0.006$ against $r=-0.271$ for $\mathrm{V} 3$ width and $r=-$ 0.260 for EDSS).

At Youden plot $(\mathrm{HV} 3>5.5)$, HV3 showed the best properties to detect low PASAT performance (sensitivity 66.1\%, specificity $81.3 \%$, PPV 92.5\%, negative predictive value 40.6\%, accuracy 70.1\%; Fig. 2).

The MS group with low PASAT performance had a significantly higher mean HV3 score $(6.81 \pm 2.34)$ than the group without impairment $(5.3 \pm 2.36 ; P=0.027)$. Conversely, for $81 \%$ of patients with MS with cognitive impairment, the HV3 score was over 5.5. 


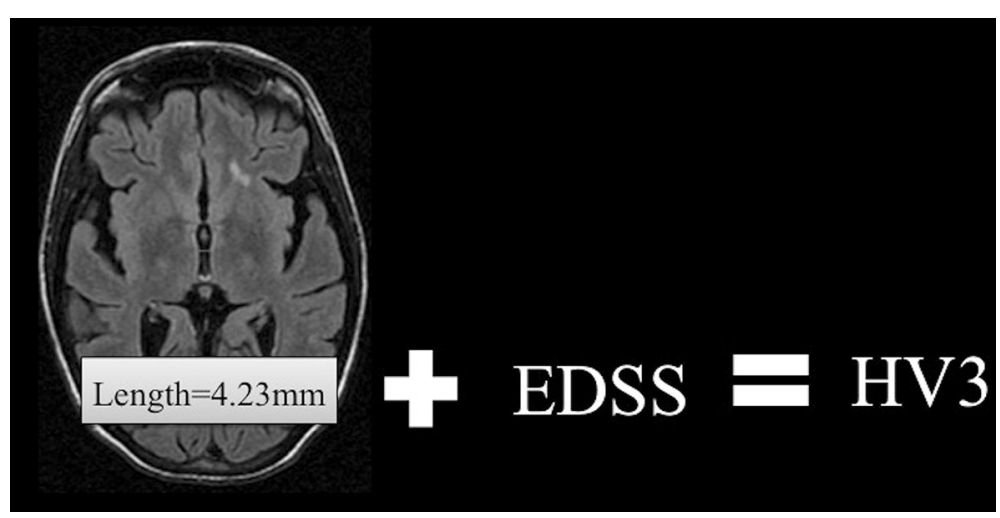

Fig. 1 The HV3 score. HV3 is obtained by summing the V3 width (in $\mathrm{mm}$; technique of determining V3 width detailed by Benedict et al. [7]) with the EDSS. EDSS Expanded Disability Status Scale, $V 3$ third ventricle

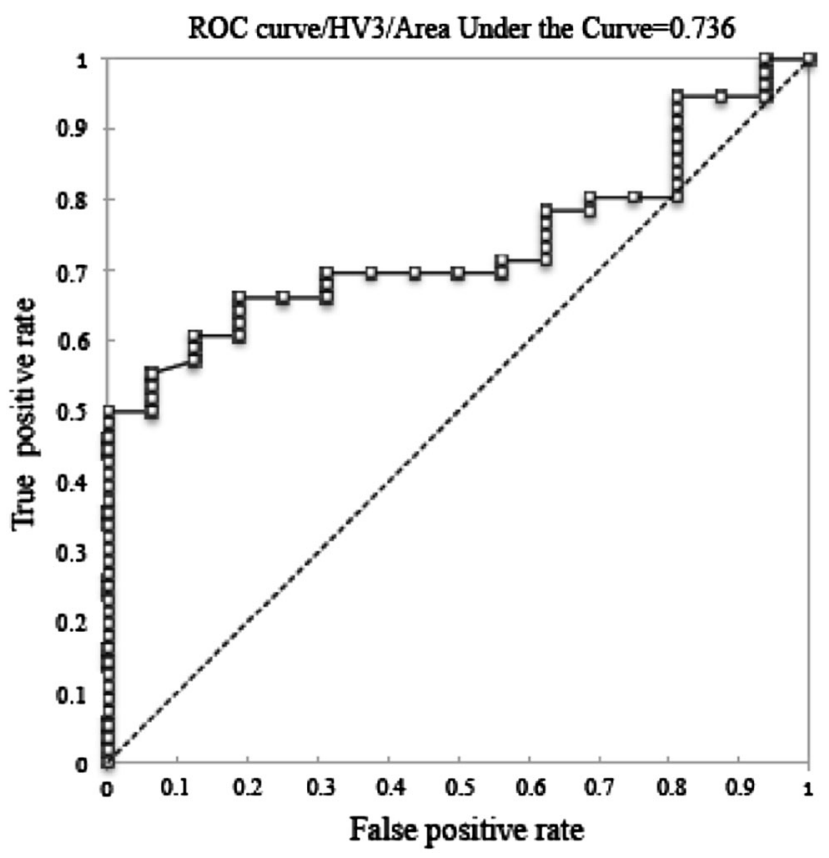

Fig. 2 Cutoff HV3 score predictive of low PASAT performance. A ROC curve was used to determine the cutoff HV3 score predicting a cognitive impairment. A score of over 5.5 was associated with low PASAT

\section{Local Atrophy}

Compared with patients with MS without cognitive impairment, right-handed patients with low PASAT performance had a more pronounced atrophy of specific brain structures: left supplementary motor area performance with a sensitivity (95\% CI) of $66.1 \%$ (53.7; $78.5)$ and a specificity $(95 \% \mathrm{CI})$ of $81.3 \%(62.1 ; 100)$. PASAT Paced Auditory Serial Addition Test, $R O C$ receiver operating characteristic

(SMA; $P=0.03$ ), inferior parietal lobules $(P<0.036)$, cingulate gyri $(P<0.039)$, and right thalamus $(P=0.027)$.

A correlation was found between the PASAT score and the local volume change of the left SMA $(r=0.295, P=0.027)$, the right parietal lobe $(r=0.277, P=0.039)$, left and right lower 
parietal lobules $(r=0.291, \quad P=0.03$ and $r=0.332, P=0.012$, respectively).

\section{DISCUSSION}

Many clinical and MRI data are associated with cognition assessment. Although the concept of cognitive impairment is defined as an alteration in at least two cognitive domains, we have chosen, in our study, to use the PASAT as a screening test for cognitive impairment. This test is generally considered a sensitive measure of cognitive dysfunction patients with MS who have mild disability [1]. It has demonstrated its ability to evaluate several cognitive domains simultaneously and is widely used because of its incorporation in the MSFC score.

As with previous studies, our study demonstrated that the V3 width was of interest as both a marker of global atrophy and cognitive decline $[7,25]$. Cognitive impairment in patients with MS may be related, at least in part, to thalamic atrophy [26]. The existence of connections between the thalamus and many brain structures involved in limbic functions and, in particular, the neocortex supports this hypothesis. Enlargement of V3 is explained by the fact that the thalami are part of the lateral wall of this structure. Our study objectified atrophy of the right thalamus in patients with MS who had a low PASAT performance. The thalamus is involved in the working memory network and is activated in functional MRI (fMRI) during the PASAT [12, 20]. Moreover, Houtchens et al. [26] showed that the EDSS was the clinical scale that correlated with thalamic atrophy. These data demonstrate that the width of the V3 was closely linked to cognitive impairment as well as disability. We also demonstrated that the left SMA, lower parietal lobules, and the cingulate gyri were more atrophied in the population with a pathological PASAT score $(P<0.05)$. These structures have been described to be activated in fMRI when performing the PASAT. Frontal lobe atrophy has been demonstrated several times in MS and is associated with cognitive impairment [11, 27, 28]. For other structures (lower parietal lobules and cingulate gyri) our study is the only one, to our knowledge, which demonstrated a link between atrophy of these structures and cognitive impairment. Audoin et al. [29] showed early atrophy of the cingulate gyri in clinically isolated syndrome but their study did not take into account cognitive impairment. Unlike other authors $[10,30]$, we did not find any significant volume variation in the callosum or in temporal lobes between patients with MS with and without cognitive impairment.

In the clinical practice, the width of the $\mathrm{V} 3$ is a structure that is workable and easily measurable by a physician using an axial FLAIR or 3D T1w sequence on brain MRI. The purpose of HV3 score is not to replace the cognitive assessment but to better identify patients requiring a full cognitive assessment. It overcomes bias linked to the evaluator, the test execution conditions, the patient's fatigue, or the retest effect, and can be done at each visit. However, with a sensitivity score of $66.1 \%$ (negative predictive value $40.6 \%$ ), while HV3 proves effective in diagnosing cognitive impairment in most patients, it is not enough to suspect all of them. Moreover, this score was defined for patients with an EDSS less than 5.5. It therefore remains to be seen whether it can be useful for patients with a more severe disability. It should be interesting to design the same study using the Symbol Digit Modalities Test or its computerized version [31-33]. 


\section{CONCLUSION}

We have established a HV3 composite score from the EDSS and the V3 width measured by MRI. From the beginning of the disease, even in patients with low T2 lesion load, deep grey matter atrophy can be detected indicating that a degenerative process is already underway. Knowing that thalamic atrophy correlates with disease progression, the use of this easy and reliable tool can be helpful for detecting progression in patients with MS, treated or not, even if they seems to be clinically asymptomatic. In these patients, the challenge is to precociously begin therapy to reduce clinical worsening. This score is an additional and complementary tool to suspect easily cognitive impairment in MS. It cannot replace a full cognitive assessment but can help to better identify patients with MS who have a risk of cognitive impairment.

\section{ACKNOWLEDGMENTS}

No funding or sponsorship was received for this study or publication of this article. All named authors meet the ICMJE criteria for authorship for this manuscript, take responsibility for the integrity of the work as a whole, and have given final approval for the version to be published.

Conflict of interest. Muriel Laffon, Grégoire Malandain, Heloïse Joly, and Mikael Cohen have no conflict of interest to declare. Christine Lebrun is a member of editorial board of Revue Neurologique, scientific board of ARSEP, OFSEP, CFSEP, IFSEP. Dr Lebrun received invitations to medical congress or boards with Biogec Idec, Merck Serono, Genzyme, Teva, Almirall, Bayer.
Compliance with ethics guidelines. The study was approved by the Nice Ethics Committee. All procedures followed were in accordance with the ethical standards of the responsible committee on human experimentation (institutional and national) and with the Helsinki Declaration of 1975, as revised in 2000 and 2008. Informed consent was obtained from all patients and controls for being included in the study.

Open Access. This article is distributed under the terms of the Creative Commons Attribution Noncommercial License which permits any noncommercial use, distribution, and reproduction in any medium, provided the original author(s) and the source are credited.

\section{REFERENCES}

1. Chiaravalloti ND, DeLuca J. Cognitive impairment in multiple sclerosis. Lancet Neurol. 2008;7:1139-51.

2. Huijbregts SCJ, Kalkers NF, de Sonneville LMJ, et al. Differences in cognitive impairment of relapsing remitting, secondary, and primary progressive MS Neurology. 2004;63:335-9.

3. Achiron A, Chapman J, Magalashvili D, et al. Modeling of cognitive impairment by disease duration in multiple sclerosis: a cross-sectional study. PLoS One. 2013;8:e71058.

4. Nocentini U, Pasqualetti P, Bonavita S, et al. Cognitive dysfunction in patients with relapsing-remitting multiple sclerosis. Mult Scler. 2006;12:77-87.

5. Benedict RHB, Fischer JS, Beatty WW, et al. Minimal neuropsychological assessment of MS patients: a consensus approach. Clin Neuropsychol. 2002;16:381-97.

6. Rao SM, Leo GJ, Bernardin L, Unverzagt F. Cognitive dysfunction in multiple sclerosis: I. Frequency, patterns, and prediction. Neurology. 1991;42:685-91.

7. Benedict RHB, Weinstock-Guttman B, Fishman I, et al. Prediction of neuropsychological impairment in multiple sclerosis: comparison of conventional magnetic resonance imaging measures of atrophy and lesion burden. Arch Neurol. 2004;61:226-30. 
8. Batista S, Zivadinov R, Hoogs M, et al. Basal ganglia, thalamus and neocortical atrophy predicting slowed cognitive processing in multiple sclerosis. J Neurol. 2011;259:139-46.

9. Calabrese M, Rinaldi F, Mattisi I, et al. Widespread cortical thinning characterizes patients with MS with mild cognitive impairment. Neurology. 2010;74:321-8.

10. Riccitelli G, Rocca MA, Pagani E, et al. Cognitive impairment in multiple sclerosis is associated to different patterns of gray matter atrophy according to clinical phenotype. 2011. Hum Brain Mapp. 2011;32:1535-43.

11. Morgen K, Sammer G, Courtney SM, et al. Evidence for a direct association between cortical atrophy and cognitive impairment in relapsing-remitting MS. Neuroimage. 2006;30:891-8.

12. Audoin B, Guye M, Reuter F, et al. Structure of WM bundles constituting the working memory system in early multiple sclerosis: a quantitative DTI tractography study. Neuroimage. 2007;36:1324-30.

13. Tona F, Petsas N, Sbardella E, et al. Multiple sclerosis: altered thalamic resting-state functional connectivity and its effect on cognitive function. Radiology. 2014;271:814-21.

14. Patti F, Amato $M$, Trojano $M$, et al. Cognitive impairment and its relation with disease measures in mildly disabled patients with relapsingremitting multiple sclerosis: baseline results from the Cognitive Impairment in Multiple Sclerosis (COGIMUS) study. Mult Scler. 2009;15:779-88.

15. Calabrese M, Agosta F, Rinaldi F, et al. Cortical lesions and atrophy associated with cognitive impairment in relapsing-remitting multiple sclerosis. Arch Neurol. 2009;66:1144-50.

16. Debouverie M, Pittion-Vouyovitch S, Louis S, et al. Validity of a French version of the fatigue impact scale in multiple sclerosis. Mult Scler. 2007;13:1026-32.

17. Gronwall DM. Paced auditory serial-addition task: a measure of recovery from concussion. Percept Mot Skills. 1977;44:367-73.

18. Reuter F, Baumstarck-Barrau K, Loundou A, et al. Paced Auditory Serial Addition Test: données normatives dans une population française. Rev Neurol (Paris). 2010;166:944-7.

19. Souplet JC, Fillard P, Toussaint N, Lebrun C, Malandain G. SepINRIA: a software to analyse multiple sclerosis brain MRI. Neurology. 2008;70:A474.
20. Bermel RA, Bakshi R, Tjoa C, et al. Bicaudate ratio as a magnetic resonance imaging marker of brain atrophy in multiple sclerosis. Arch Neurol. 2002;59:275-80.

21. Simon JH, Jacobs LD, Campion MK, et al. A longitudinal study of brain atrophy in relapsing multiple sclerosis. The Multiple Sclerosis Collaborative Research Group (MSCRG). Neurology. 1999;53:139-48.

22. Rosset A, Spadola L, Ratib O. OsiriX: an open-source software for navigating in multidimensional DICOM images. J Digit Imaging. 2004;17:205-16.

23. Guimond A. Average brain models: a convergence study. Comput Vis Image Underst. 2000;77:192-210.

24. Audoin B, Ibarrola D, Duong MVA, et al. Functional MRI study of PASAT in normal subjects. MAGMA. 2005;18:96-102.

25. Muller M, Esser R, Kotter K, et al. Third ventricular enlargement in early stages of multiple sclerosis is a predictor of motor and neuropsychological deficits: a cross-sectional study. BMJ Open. 2013;3:e003582.

26. Houtchens MK, Benedict RHB, Killiany R, et al. Thalamic atrophy and cognition in multiple sclerosis. Neurology. 2007;69:1213-23.

27. Benedict RHB, Bakshi R, Simon JH, et al. Frontal cortex atrophy predicts cognitive impairment in multiple sclerosis. J Neuropsychiatry Clin Neurosci. 2002;14:44-51.

28. Audoin B, Davies GR, Finisku L, et al. Localization of grey matter atrophy in early RRMS. J Neurol. 2006;253:1495-501.

29. Audoin B, Zaaraoui W, Reuter F, et al. Atrophy mainly affects the limbic system and the deep grey matter at the first stage of multiple sclerosis. J Neurol Neurosurg Psychiatry. 2010;81:690-5.

30. Pelletier J, Suchet L, Witjas T, et al. A longitudinal study of callosal atrophy and interhemispheric dysfunction in relapsing-remitting multiple sclerosis. Arch Neurol. 2001;58:105-11.

31. Deloire M, Bonnet M, Salort E, et al. How to detect cognitive dysfunction at early stages of multiple sclerosis? Mult Scler. 2006;12:445-52.

32. Benedict RH, Amato MP, Boringa J, et al. Brief International Cognitive Assessment for MS (BICAMS): international standards for validation. BMC Neurol. 2012;12:56.

33. Ruet A, Deloire MS, Charre-Morin J, et al. A new computerised cognitive test for the detection of information processing speed impairment in multiple sclerosis. Mult Scler. 2013;19:1665-72. 